\title{
Numerical study for production of space charge within the stratiform cloud
}

\author{
A K Srivastava,* and S N Tripathi** \\ Department of Civil Engineering, Indian Institute of Technology Kanpur, Kanpur 280 016, India. \\ ${ }^{1}$ Current address: Indian Institute of Tropical Meteorology (Branch), Prof Ramnath Vij Marg, \\ New Delhi, India. \\ *e-mail: atul@tropmet.res.in \\ **e-mail:snt@iitk.ac.in
}

\begin{abstract}
One dimensional numerical model has been developed to predict the production of space charge and variations in other electrical parameters within the low level stratiform type of cloud having very weak vertical motion. Non-linear coupled differential equations which govern ion concentrations, charged and neutral droplet concentrations and electric field were used. Symmetry has been observed in all the electrical parameters within the cloud. The magnitude of average positive ion concentrations was observed to be high as compared to the negative ion concentrations, which is due to low scavenging rate of positive ions than the negative ions, highly attributed to their mobilities. The rate of scavenging of ions affects the concentration of charged droplets, which eventually influence the electric field and thus the space charge density within the cloud. Maximum electric field $\left(E_{\max }\right)$ was observed at middle of the cloud whereas minimum was observed at both the edges of the cloud. Minimum electric field $\left(E_{\min }\right)$ was found to be equal and constant $\left(\sim 27 \mathrm{Vm}^{-1}\right)$ for any drop concentration. Net positive and negative space charges were observed at the top and bottom of the cloud, respectively. The simulated results show some discrepancies to the natural condition, which are due to simulations made under some basic assumptions and limitations and that will be incorporated in the future studies for natural cloud condition.
\end{abstract}

\section{Introduction}

The Earth's climate could be affected by changes in cloudiness caused by variations in the intensity of galactic cosmic rays (GCRs) in the atmosphere (Svensmark and Friis-Christebsen 1997; Carslaw et al 2002; Siingh and Singh 2009 with references therein). The effect of cosmic rays on climate could be in three ways:

- through changes in the concentration of cloud condensation nuclei,

- thunderstorm electrification, and

- ice formation in cyclones.

The concentration of cloud condensation nuclei depends on the light ions produced during cosmic ray ionization (Farrar 2000; Carslaw et al 2002). Svensmark et al (2007) based on a laboratory experiment in which a gas mixture equivalent to chemical composition of the lower atmosphere was subjected to UV light and cosmic rays, reported that the released electrons promoted fast and efficient formation of ultra-fine aerosol particles which may grow to become cloud condensation nuclei. Aircraft-based ion mass spectrometer measurements in the upper troposphere have shown an evidence for cosmic ray induced aerosol formation (Solanki 2002; Mouel et al 2005). Observational studies show that the cosmic ray and cloud effect seems to be present largely in the low-altitude clouds, because low-altitude clouds exert a large net cooling effect on the climate;

Keywords. Stratiform cloud; space charge; electric field; attachment coefficient. 
this determines the sign of the possible cosmic ray and cloud effect (Svensmark and Christensen 1997; Marsh and Svensmark 2000). Although the lowaltitude clouds of limited vertical extent may only contain one phase of liquid water, it is a key parameter in the numerical climate models used for future climate change predictions. Kirkby (2007) had presented an association of high GCR flux with cooler climate, and low GCR flux with a warmer climate.

Clouds having both convective and stratiform types have different electrical properties. To date several numerical schemes have been constructed to understand the electrification and the production of space charges within the convective clouds, which have strong vertical motion and strong electric field (Chiu and Klett 1976; Masuelli et al 1997; Helsdon et al 2001; Altaratz 2003; Saunders 2008). However, no numerical studies, to the best of our knowledge, have been carried out to understand the production of space charge within the low level stratiform type of cloud having very weak vertical motion and weak electric field inside it.

Electrical structures of weakly electrified clouds such as stratus are less well known, which has been a subject of investigation (Tinsley et al 2000; Harrison and Carslaw 2003; Tinsley and Yu 2004). Stratus is a low level stratiform cloud mainly composed of water droplets with little vertical thickness but tend to be spread out into a thin sheet, having maximum thickness of about $1 \mathrm{~km}$ from top to bottom and large aerial coverage. It has been conjectured that inside the clouds, where conductivity reduces by about an order of magnitude with respect to clear air (Rust and Moore 1974), large space charge (up to few tens to thousands elementary charges per $\mathrm{cm}^{-3}$ ) can accumulate (Tinsley 2000). The space charge, mostly the difference between uni-polar charges, is implicated for droplet charging by various processes notably amongst them is preferential scavenging of aerosol particles (electro-scavenging) (Tinsley 2000). The space charge increases with the electric field in the low-conductivity region within the cloud, thereby restoring the equilibrium vertical conduction current. Thus, although electrical effects are much weaker in stratiform clouds than in convective, they are certainly present and the electric fields and charge densities are modulated by cosmic rays (Carslaw et al 2002).

In this paper, we have made an attempt to develop a model for the production of space charge and to understand the electrical parameters, such as electric field within the low level stratiform cloud using non-linear coupled differential equations, which govern the ion concentration, charged and neutral droplet concentration and electric field variation inside the cloud. The detailed model

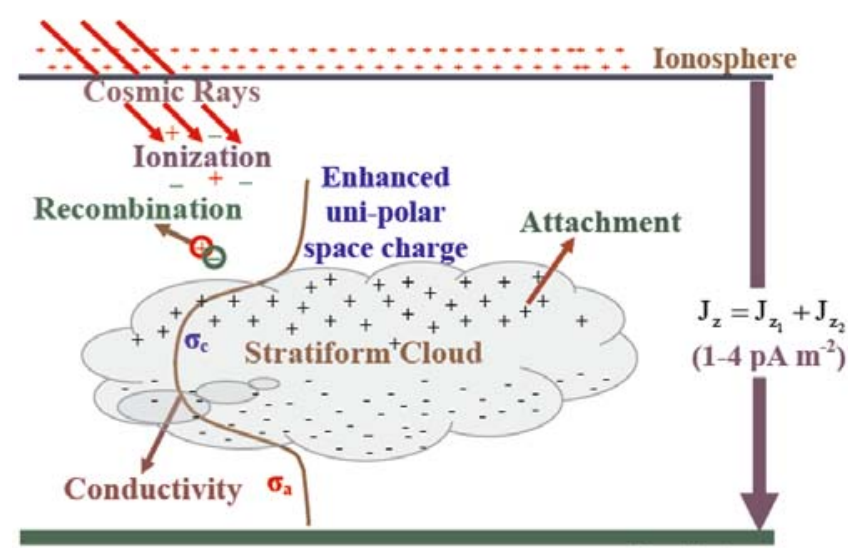

Earth Surface

Figure 1. Schematic diagram of production of uni-polar space charge due to processes occurring in the presence of cosmic rays along with the variation in conductivity within the stratiform cloud. Total air-Earth's current density $\left(J_{z}\right)$, penetrating from top of the cloud, is considered constant with height.

description along with assumptions and initial boundary conditions are given in the next section.

\section{Model descriptions}

\subsection{Physical understanding}

Stratiform clouds could be electrified because their presence in the free atmosphere reduces conductivity (Rust and Moore 1974; Pruppacher and Klett 1997) and the influence of space charge accumulation on their boundaries due to variations in air-Earth's current density (Tinsley 2000), causing changes in cloud microphysics. Figure 1 shows a schematic diagram based on the hypothesis proposed by Tinsley (2000) for the production of uni-polar space charge within weakly electrified stratiform type of cloud. It shows total air-Earth's current density $\left(J_{z}=J_{z_{1}}+J_{z_{2}}\right)$, a product of conductivity and electric field, penetrating from top of the cloud, is constant with height ranging from $\sim 1-4 \mathrm{pA} \mathrm{m}^{-2}$ into and out of each volume. However, $J_{z_{1}}$ (positive current density) and $J_{z_{2}}$ (negative current density) vary with height due to recombination and attachment of ions that occur in the same volume. Thus, the regions of net unipolar space charge often exist on the upper and lower boundaries of the cloud. Schematic diagram also shows variation in conductivity that reduces within the cloud $\left(\sigma_{c}\right)$ as compared to the clear air $\left(\sigma_{a}\right)$ due to scavenging of ions by their attachment with cloud droplets.

\subsection{Theoretical formulation}

A 1-D numerical model, to study the production of space charges and other electrical properties such 
as electric field, has been developed for low level stratiform clouds having weak electric field and weak vertical motion. The non-linear system has been modeled using coupled differential equations for the rate of change of ion concentration, charged and neutral droplet concentration and electric field variation with height.

The time dependent conservation equation for positive ions represented by 1 and negative ions represented by 2 is given as:

$$
\begin{aligned}
& \frac{d n_{1}}{d t}=q-\alpha n_{1} n_{2}-\beta_{1}\left(S n_{1}+S_{2} n_{1}\right)-\frac{1}{e} \nabla J_{z_{1}}, \\
& \frac{d n_{2}}{d t}=q-\alpha n_{1} n_{2}-\beta_{2}\left(S n_{2}+S_{1} n_{2}\right)+\frac{1}{e} \nabla J_{z_{2}} .
\end{aligned}
$$

Here $n_{1,2}$ are positive (negative) ion concentration, $q$ is the ion production rate, $\alpha$ is the ion-ion recombination coefficient, $\beta_{1,2}$ are positive (negative) ion attachment coefficients with droplets, $S_{1,2}$ are charged droplet concentrations having unit positive (negative) charge, $S$ is the neutral droplet concentrations, $e$ is the electronic charge and $J_{z_{1,2}}$ is vertical current density.

The above two equations are balanced by the production of ions due to ionization from cosmic rays, i.e., $q$ and loss of ions by various mechanism such as ion-ion recombination, which play a crucial role in ultra-fine particle formation in the lower atmosphere (Mohnen 1977; Yu and Turco 2001), and ion-droplet attachment within the cloud (Griffiths et al 1974). The last term of equations (1) and (2) indicates flux of positive $\left(\nabla J_{z_{1}}\right)$ and negative $\left(\nabla J_{z_{2}}\right)$ ions, respectively which produces an imbalance between both the ions in a region. Direct measurement of total current density, $J_{z}$ is difficult, therefore it is usually derived from the simultaneous measurements of conductivity and electric field and can be expressed as:

$$
J_{z}=J_{z_{1}}+J_{z_{2}}=\sigma_{z_{1}} E_{z}+\sigma_{z_{2}} E_{z} .
$$

By such an approach, $J_{z}$ is found to be constant vertically in the stable and fair weather conditions (Gringel et al 1986; Siingh et al 2007 and the references therein). In equation (3), $\sigma_{z_{1}}$ and $\sigma_{z_{2}}$ are the positive and negative ion conductivity, respectively which can be expressed in terms of ionic number density $\left(n_{1}, n_{2}\right)$ and mobility of ions $\left(\mu_{z 1}, \mu_{z 2}\right)$. The total current density from equation (3) becomes:

$$
J_{z}=\left(\mu_{z_{1}} n_{1} e+\mu_{z_{2}} n_{2} e\right) E_{z} ;
$$

$$
E_{z}=\frac{J_{z}}{e}\left(\frac{1}{\mu_{z_{1}} n_{1}+\mu_{z_{2}} n_{2}}\right) .
$$

Equation (4) can be used as an initial value in the model for the electric field at cloud boundaries using total current density $\left(J_{z}\right)$ penetrating from top of the cloud.

Now, the last term of equations (1) and (2) can be written as:

$$
\begin{aligned}
& \frac{1}{e} \nabla J_{z_{1}}=\frac{1}{e} \nabla\left(\mu_{z_{1}} n_{1} e E_{z}\right) ; \\
& \frac{1}{e} \nabla J_{z_{2}}=\frac{1}{e} \nabla\left(\mu_{z_{2}} n_{2} e E_{2}\right) .
\end{aligned}
$$

For horizontal stratification and at steady state, the above equations can be written as:

$$
\begin{aligned}
& \frac{1}{e} \nabla J_{z_{1}}=\frac{1}{e} \frac{\partial}{\partial z}\left(J_{z_{1}}\right)=\mu_{z_{1}} n_{1} \frac{\partial}{\partial z}\left(E_{z}\right)+E_{z} \frac{\partial}{\partial z}\left(\mu_{z_{1}} n_{1}\right) \\
& \frac{1}{e} \nabla J_{z_{2}}=\frac{1}{e} \frac{\partial}{\partial z}\left(J_{z_{2}}\right)=\mu_{z_{2}} n_{2} \frac{\partial}{\partial z}\left(E_{z}\right)+E_{z} \frac{\partial}{\partial z}\left(\mu_{z_{2}} n_{2}\right) .
\end{aligned}
$$

Using the values from equation (6), equations (1) and (2) becomes:

$$
\begin{aligned}
\frac{d n_{1}}{d t}= & q-\alpha n_{1} n_{2}-\beta_{1}\left(S n_{1}+S_{2} n_{1}\right) \\
& -\mu_{z_{1}} n_{1} \frac{\partial}{\partial z}\left(E_{z}\right)-E_{z} \frac{\partial}{\partial z}\left(\mu_{z_{1}} n_{1}\right) ; \\
\frac{d n_{2}}{d t}= & q-\alpha n_{1} n_{2}-\beta_{2}\left(S n_{1}+S_{2} n_{1}\right) \\
& +\mu_{z_{2}} n_{2} \frac{\partial}{\partial z}\left(E_{z}\right)+E_{z} \frac{\partial}{\partial z}\left(\mu_{z_{2}} n_{2}\right) .
\end{aligned}
$$

$S_{1}$ and $S_{2}$ are the number concentration of singly positive and negative charge droplets, respectively. The time dependent balance equation for charged particles is depicted by Harrison and Carslaw (2003). However, by ignoring positive ions attachment to the positively charged droplets and negative ions attachment to the negatively charged droplets, the balance equations for single positive and single negative charge drops can be written in equations (8) and (9), respectively as:

$$
\frac{d S_{1}}{d t}=\beta_{1} S n_{1}-\beta_{2} S_{1} n_{2} ;
$$




$$
\frac{d S_{2}}{d t}=\beta_{2} S n_{2}-\beta_{1} S_{2} n_{1}
$$

The first term of both equations represent the production of positively and negatively charged droplets due to the attachment of positive and negative ions with neutral droplets, respectively whereas the last term represents loss of positively and negatively charged droplets due to their attachment with negative and positive ions, respectively. $S$ is the neutral droplet concentration within the cloud, which can also be written by using above two equations (8) and (9) as:

$$
\frac{d S}{d t}=\left(\beta_{2} S_{1} n_{2}+\beta_{1} S_{2} n_{1}\right)-\left(\beta_{1} S n_{1}+\beta_{2} S n_{2}\right)
$$

The first bracket indicates the production of neutral droplets by neutralizing the positively and negatively charged droplets due to their attachment with negative and positive ions, respectively. However, the second bracket represents loss of neutral droplets due to their attachment with positive and negative ions, respectively.

The characteristic of ions that relates directly to their ability to take part in the flow of electric current, is their mobility $(\mu)$, which is the average velocity acquired by small ions as it moves under the force exerted on it by the electric field. Ion mobility controls the rate of uptake of ions by the aerosols and droplets. Mobility varies inversely with the mass of cluster of ions (Harrison 1992);

$$
\mu=\frac{2 \times 10^{-4}}{\sqrt{(M+29 / M)}}
$$

where $M$ is the mass of ions.

In the present cloud model, $\mathrm{H}^{+}\left(\mathrm{H}_{2} \mathrm{O}\right)_{m}$ is used as positive ion $\left(\mu_{1}\right)$ and $\mathrm{O}_{2}^{-}\left(\mathrm{H}_{2} \mathrm{O}\right)_{n}$ as negative ion $\left(\mu_{2}\right)$ where ' $m$ ' and ' $n$ ' are the hydrated water molecules, vary in such a manner that both the ion mobilities are maximum at the edges of the cloud and decreasing inside with minimum at the middle of the cloud, as shown in figure 2 .

The chemical differences between the species of positive and negative ions lead to some physical asymmetries in the ion properties and cause negative ions to have greater mobility than positive ions, i.e., $\mu_{2}>\mu_{1}$ (Mohnen 1977). The magnitude of the difference is typically $\sim 20 \%$ but varies with humidity in response to changes in the ion hydration as well as ion composition (Harrison and Carslaw 2003). This asymmetry is central to the cloud electrification. In the present study, average positive ion mobility was observed to be $\sim 7 \%$ less



Figure 2. Variation in positive $\left(\mu_{1}\right)$ and negative $\left(\mu_{2}\right)$ ion mobilities inside the cloud.

as compared to the average negative ion mobility within the cloud. The positive and negative ion mobility at the cloud boundaries were calculated to be maximum $\sim 1.3 \times 10^{-4}$ and $1.5 \times 10^{-4} \mathrm{~m}^{2} \mathrm{~V}^{-1} \mathrm{~s}^{-1}$ using low hydrated water molecules, i.e., $m=4$ and $n=1$, respectively which approaches to minimum $\sim 1.0 \times 10^{-4}$ and $9.75 \times 10^{-5} \mathrm{~m}^{2} \mathrm{~V}^{-1} \mathrm{~s}^{-1}$ using large hydrated water molecules, i.e., $m=6$ and $n=3$, respectively at middle of the cloud. The difference between both the ionic mobilities are high at the boundaries where negative ion mobility was $\sim 15 \%$ more than the positive ion mobility and low at the middle of the cloud where negative ion mobility was $\sim 4 \%$ more than the positive ion mobility. To get the mobility values at each point within the cloud, a second order polynomial was fitted for both the ionic mobilities with step size $(X)$ and the polynomial equations are:

$$
\begin{aligned}
Y= & 1.20648 \times 10^{-4}-9.30144 \times 10^{-8} \\
& \times X+9.30144 \times 10^{-11} \times X^{2}, \\
Y= & 1.34547 \times 10^{-4}-1.34673 \times 10^{-7} \\
& \times X+1.34673 \times 10^{-10} \times X^{2} .
\end{aligned}
$$

Equations (12a) and (12b) predict a decrease in both positive and negative ion mobilities by $\sim 25 \%$ and $33 \%$, respectively from cloud boundaries to the middle of the cloud.

The rate of uptake of ions by cloud droplets is known as collision rate, which leads to charging of these droplets. It is normally expressed in terms of attachment coefficient, which varies with height as a function of ionic mobility. The attachment coefficient, primarily, depends on droplet size and the number concentration of ions of both polarities. 
It is also said to depend on the magnitude of charges carried by the droplets. However, when cloud droplets act as collectors of ions, the collection current is independent of the droplet charge for typical conditions (Pruppacher and Klett 1997, pp 798). So, the charged droplets of either sign and the neutral droplets should all collect ions at the same rate that only depends on the properties of the ions. Attachment coefficients, $\beta_{1,2}$ for positive (negative) ions with cloud droplet of radii ' $a_{c}$ ' were given by Griffiths et al (1974); Chiu (1978); Pruppacher and Klett (1997):

$$
\beta_{1,2}\left(a_{c}\right)=4 \pi a_{c} D_{1,2}
$$

where $D_{1,2}=\left(\mu_{1,2} K T / e\right)$ is the diffusion coefficient of positive (negative) ions.

Thus, equation (14) becomes:

$$
\beta_{1,2}\left(a_{c}\right)=\frac{4 \pi a_{c} K T \mu_{1,2}}{e}
$$

where $K$ is the Boltzmann constant and $T$ is the temperature.

The radius of cloud droplets of average size ' $a_{c}$ ' can be computed from a relationship given by Chiu (1978):

$$
a_{c}=\left(\frac{3}{4} \frac{\rho l_{c}}{\pi \rho_{w} S}\right)^{1 / 3}
$$

where $\rho$ is the density of air $\left(\sim 1.239 \mathrm{~kg} \mathrm{~m}^{-3}\right), l_{c}$ is the liquid water content within the cloud, $\rho_{w}$ is the density of water $\left(\sim 1000 \mathrm{~kg} \mathrm{~m}^{-3}\right)$ and $S$ is the drop concentration within the cloud. Equation (16) reveals that the average drop radii will decrease with increase in drop number concentration within the cloud.

The majority of free charges in the fair weather part of the atmosphere arise from natural ionization, e.g., cosmic rays (Chalmers 1967; Siingh and Singh 2009), which is more dominant activity away from the surface. The space charge, in the fair weather, is the difference between the concentration of positive and negative ions. However, within the clouds most of the positive and negative ions will be in the form of charged droplets (Tinsley 2000). Therefore, space charge, in the above case, will be the sum of differences between the concentration of positive and negative ions and the concentration of positively and negatively charged droplets, which is given as:

$$
\rho=e\left[\left(n_{1}-n_{2}\right)+\left(S_{1}-S_{2}\right)\right] .
$$

Variations in electric field $(E)$ and space charge $(\rho)$ are coupled by Poisson's equation $(\nabla \cdot E=(\rho / \varepsilon))$; where $\varepsilon$ is the permittivity of vacume $\left(\sim 8.85419 e-12 \mathrm{~F} \mathrm{~m}^{-1}\right)$. Thus, assuming horizontal stratification, the Poisson's equation can be written as:

$$
\frac{\partial E_{z}}{\partial z}=\frac{\rho}{\varepsilon}=\frac{e}{\varepsilon}\left[\left(n_{1}-n_{2}\right)+\left(S_{1}-S_{2}\right)\right]
$$

To develop a 1-D numerical model to understand the electrical properties and the production of space charge inside the stratiform cloud, we have solved equations (7a), (7b), (8), (9), (10) and (17) simultaneously, using Gauss-Seidel iterative method (Jain 1984) to get solution for enough time to come to a steady state. The methodology of solving these equations will be discussed in the next section, which also includes basic assumptions and the boundary conditions of the model.

\subsection{Methodology}

The above equations were discretized using purely implicit finite difference scheme, which enables the integration of a differential equation numerically by evaluating the values of the function at finite number of points. All the equations were integrated for sufficient time until steady state condition is reached. Finite difference scheme, applied here, has been solved using false transient method in time step, i.e., the steady state time computed by the program is independent of actual time. The origin of this method is Taylor series expansion, which assumes that the function is smooth, i.e., continuous and differentiable. Time derivative is discretized using forward difference and space derivatives with backward/forward difference usually called forward time backward space (FTBS) and/or forward time forward space (FTFS) method of discretization (Ghoshdastidar 1998).

The model presented here is for stable cloud layer, i.e., no vertical or horizontal motions exist within the cloud. The basic assumption made while solving these equations is: cloud contains monodispersed droplets (i.e., all with the same radius), representing the dominant size category, with at most a single positive and/or negative charge. Thus, $\beta$ (ion attachment coefficient with droplet) in each case apply to the radius of the dominant droplets. The boundary conditions of the model includes:

- equal and maximum positive and negative ion concentration, 
Table 1. Typical values of the atmospheric parameters used to calculate the initial boundary conditions of model.

\begin{tabular}{ccc}
\hline $\begin{array}{c}\text { Atmospheric } \\
\text { components }\end{array}$ & Typical values & \multicolumn{1}{c}{ References } \\
\hline$q$ & $\sim 1 \times 10^{7}$ ion & Schonland (1953) \\
& pair m $\mathrm{m}^{-3} \mathrm{~s}^{-1}$ & \\
$\alpha$ & $\sim 1.6 \times 10^{-12} \mathrm{~m}^{3} \mathrm{~s}^{-1}$ & Schonland (1953) \\
$J_{z}$ & $\sim 3 \mathrm{pA} \mathrm{m}^{-2}$ & Harrison (2005) \\
\hline
\end{tabular}

Table 2. Average drop concentrations and the corresponding radii within the cloud for a constant liquid water content $\sim 0.3 \mathrm{~g} \mathrm{~kg}^{-1}$ within stratus cloud (Chiu 1978).

\begin{tabular}{cc}
\hline $\begin{array}{c}\text { Average drop } \\
\text { concentration }\left(\mathrm{m}^{-3}\right)\end{array}$ & $\begin{array}{c}\text { Drop radii } \\
(\mu \mathrm{m})\end{array}$ \\
\hline $1.0 \mathrm{e} 8$ & 9.5 \\
$5.0 \mathrm{e} 8$ & 5.6 \\
$1.0 \mathrm{e} 9$ & 4.5 \\
\hline
\end{tabular}

- total drop concentration are zero at top and bottom of cloud, consequently,

- total charged and neutral droplet concentration are also zero at both the cloud boundaries.

$$
\begin{aligned}
& n_{1,2}(0, t)=n_{1,2}(N, t)=\left(\frac{q}{\alpha}\right)^{1 / 2}, \\
& E(0)=E(N)=\frac{J_{z}}{e}\left(\mu_{1} n_{1}+\mu_{2} n_{2}\right), \\
& S_{1,2}(0, t)=S_{1,2}(N, t)=0, \\
& S(0, t)=S(N, t)=0,
\end{aligned}
$$

where ' 0 ' and ' $N$ ' represents first and last boundary of the cloud. $S$ is the total drop concentration when they are uncharged at time $t=0$, which we have assumed to be varied sinusoidally within the cloud as $S=S_{0}(\sin (\pi \Delta z / Z))^{2}$ with maximum concentration at middle of the cloud. $S_{0}$ is the maximum drop concentration at middle of the cloud; $Z$ is the total thickness of the cloud $(1000 \mathrm{~m}$ in the present simulation) and $\Delta z$ is the small increment $(1 \mathrm{~m})$. Typical value of the atmospheric parameters, used to calculate the initial boundary conditions for ion concentration and electric field, is given in table 1.

\section{Results}

The variations in ionic concentration, within the cloud, are extensively attributed to the variations

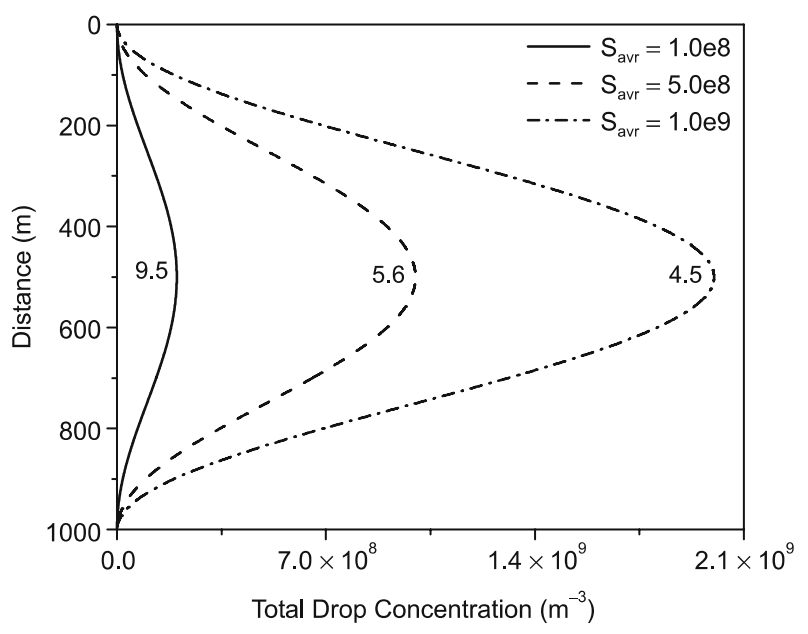

Figure 3. Variation in total drop concentrations within the cloud at different drop sizes.

in drop number concentration and their sizes through scavenging due to attachment process that influence the concentration of charged droplets and thus the production of space charge and electric field (Harrison and Carslaw 2003). The experimentally determined cloud droplet number concentrations are to be below $1.0 \mathrm{e} 8 \mathrm{~m}^{-3}$ and rarely above $1.0 \mathrm{e} 9 \mathrm{~m}^{-3}$ within the stratiform clouds (Anderson et al 1994; Gillani et al 1995). In the present study, we have considered three different sets of calculation assuming three different average drop concentrations $1.0 \mathrm{e} 8,5.0 \mathrm{e} 8$ and $1.0 \mathrm{e} 9 \mathrm{~m}^{-3}$ having mono-dispersed drops of average drop radii $\sim 9.5$, 5.6 and $4.5 \mu \mathrm{m}$, respectively, which were calculated from equation (16) keeping constant liquid water content $\sim 0.3 \mathrm{gm} \mathrm{kg}^{-1}$ within stratus cloud (Chiu 1978) and shown in table 2.

\subsection{Total drop concentration}

The drop concentration within the cloud is one of the important parameter, which influences the cloud electrification. The variation in total drop concentrations within the cloud having three different drop sizes (mono-dispersed) are shown in figure 3, which indicates maximum drop concentration at middle of the cloud. It also reveals that the large average drop concentrations are having smaller drop sizes and vice-versa.

\subsection{Total ion concentration}

The variation in ion concentrations within the cloud is shown in figure 4 for three different average drop concentrations, as mentioned before. The magnitude of average positive and negative ion concentrations were observed to be $\sim 8.18 \mathrm{e} 8$ and $8.16 \mathrm{e} 8 \mathrm{~m}^{-3}$, respectively within the cloud for drop concentration $1.0 \mathrm{e} 8 \mathrm{~m}^{-3}$ having drop radii 

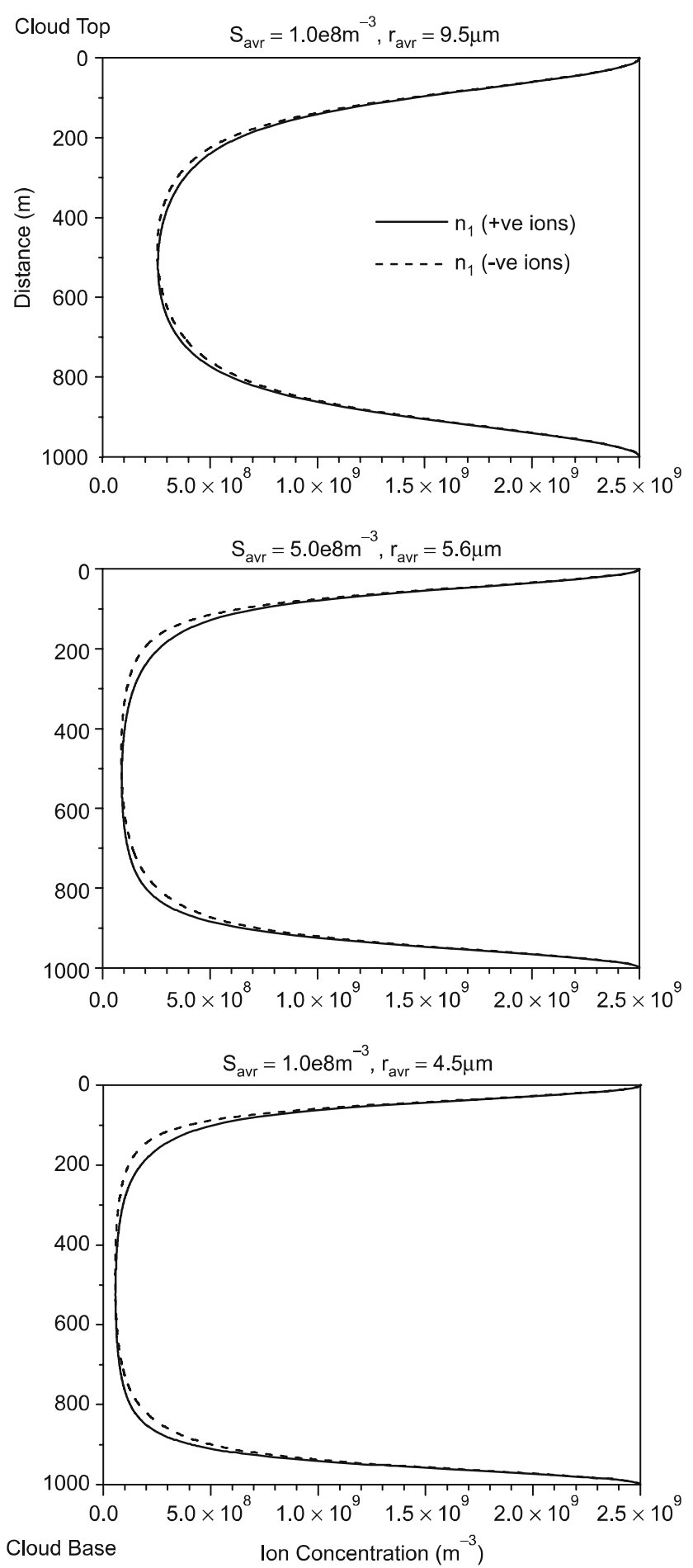

Figure 4. Variation in ionic concentrations within the cloud having different drop concentrations at different sizes.

$\sim 9.5 \mu \mathrm{m}$. Significant decrease in bi-polar ionic concentrations was observed by $\sim 42 \%$ and $54 \%$ as the drop concentrations increased by 5 and 10 times with significant decrease in drop radii $\sim 41 \%$ and $52 \%$, respectively. In all the three cases, the average positive ion concentrations were observed to be higher than the negative ion concentrations within the cloud, which is due to smaller attachment coefficient of positive ions as compared to the negative ions.

The attachment coefficient of ions to the cloud drops highly depend on the mobility of ions, drop sizes and also on the drop concentrations (equations 14 and 15). It generally decreases with decreasing drop sizes (not shown here). The rate of ion removal or scavenging within the cloud highly depends on their attachment coefficients and drop concentrations apart from drop size. Thus, the results obtained (figure 4) reveal a decrease in ion scavenging for larger size drops $(\sim 9.5 \mu \mathrm{m})$, which is mainly attributed to the presence of significantly lower drop concentrations $\left(1.0 \mathrm{e} 8 \mathrm{~m}^{-3}\right)$. Therefore, although the attachment coefficient is high, in the above case, ion scavenging rate is low. Figure also shows a significant increase in ion scavenging rate due to increased average drop concentrations by 5 and 10 times with significant decrease in drop radii $\sim 41 \%$ and $52 \%$, respectively.

The concentration of both ions decreased up to about middle of the cloud where it crosses each other, above which the average positive ion concentrations were observed to be $\sim 3 \%, 9 \%$ and $12 \%$ more than that of the average negative ion concentrations having average drop concentrations $1.0 \mathrm{e} 8,5.0 \mathrm{e} 8$ and $1.0 \mathrm{e} 9 \mathrm{~m}^{-3}$ with radii $\sim 9.5,5.6$ and $4.5 \mu \mathrm{m}$, respectively; however, opposite was true near the bottom of the cloud. In this case, higher positive ions near the top and higher negative ions near the bottom of the cloud were observed due to unequal loss rates of two ions. Production of both ions are same but loss of negative ions is more near the cloud top and vice-versa is true near the cloud bottom.

\subsection{Total charged and neutral drop concentration}

Because total drop concentrations within the cloud are considered to vary sinusoidally with altitude, it is maximum at the middle and minimum at the cloud boundaries (shown in figure 3 for different size of droplets). Consequently, the ion concentration will significantly reduce up to middle of the cloud due to their attachment to the cloud droplets, as already discussed in figure 4 . The ion removal process will thus increase the charged drop concentrations, i.e., positive $\left(S_{1}\right)$ and negative charge drops $\left(S_{2}\right)$ as shown in figure 5 with dash and dashdot lines, respectively, and consequently decrease in total drop concentrations $(S)$ within the cloud at steady-state. The steady-state uncharged droplets are termed as neutral droplet concentrations $\left(S_{o}\right)$, which is shown in the same figure with solid line. Average positive, negative and neutral drop concentrations were observed $\sim 32,35$ and $33 \%$ of the total drop concentrations. 



Figure 5. Variation in positively charged, $S_{1}$ (dash line), negatively charged, $S_{2}$ (dash-dot line) and neutral, $S_{o}$ (solid line) drop concentrations within the cloud having different drop concentrations of different sizes.

In contrast to the average positive and negative ion concentrations within the cloud, average positive charge drops were observed to be less as compared to the average negative charge drops. This is due to the low magnitude of attachment coefficient of positive ions as compared to that of negative ions, as discussed before. The magnitude of average

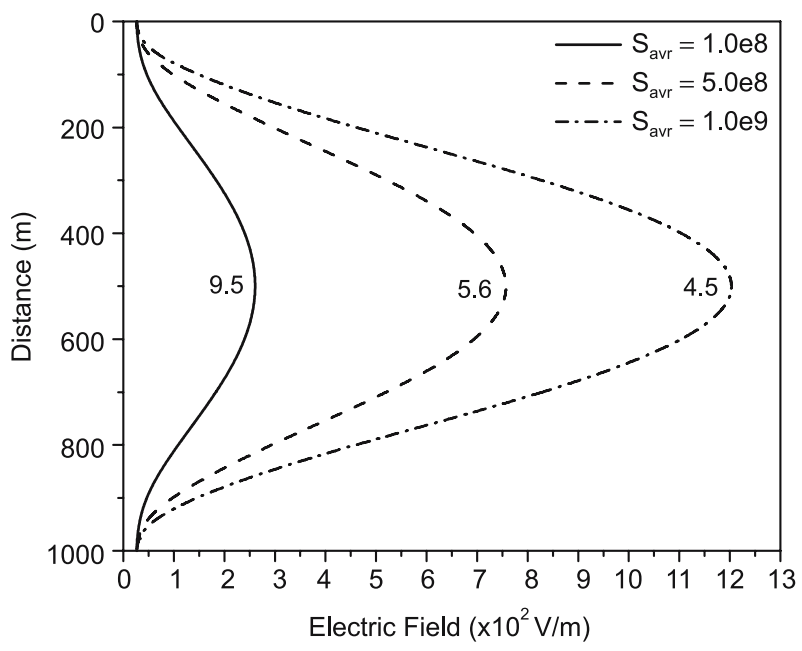

Figure 6. Variation in electric field within the cloud having different drop concentrations at different sizes.

positive and negative charged drops were observed $\sim 3.2 \mathrm{e} 7$ and $3.5 \mathrm{e}^{-3} \mathrm{~m}^{-3}$ within the cloud, containing average drop concentrations $1.0 \mathrm{e} 8 \mathrm{~m}^{-3}$ having drop radii $\sim 9.5 \mu \mathrm{m}$, which increases by $\sim 5$ and 10 times as the average drop concentrations increased by 5 and 10 times with significant decrease in drop radii $\sim 41 \%$ and $52 \%$, respectively. The concentration of both the charged droplets were observed to be increased up to about middle of the cloud where it crosses each other and showing similar behaviour as obtained in case of ionic concentrations near top and bottom of the cloud. The remaining uncharged droplets, termed as neutral, were also observed within the cloud, which compensate the total drop concentrations.

\subsection{Electric field}

An enhancement in the production of charged droplets associated with ion removal will consequently increase the electric field within the cloud to compensate vertical current density, as shown in figure 6. Minimum electric field $\left(E_{\min }\right)$ was observed $\sim 27 \mathrm{~V} \mathrm{~m}^{-1}$ at both the edges of cloud, which is the constant initial value goes into the model, calculated using various initial parameters already discussed earlier. Maximum electric field $\left(E_{\max }\right)$ was observed at middle of the cloud where it was $\sim 260 \mathrm{~V} \mathrm{~m}^{-1}$ in the case of cloud having average drop concentrations $1.0 \mathrm{e} 8 \mathrm{~m}^{-3}$ with radii $\sim 9.5 \mu \mathrm{m}$. It increases significantly by $\sim 3$ and 5 times as the average drop concentrations increased by 5 and 10 times with significant decrease in drop radii $\sim 41 \%$ and $52 \%$, respectively. Variations in electric field inside the cloud are in accordance with the observed electric field variations in nonthunderstorm cloud as reported by MacGorman and Rust (1998, pp. 46-47). 


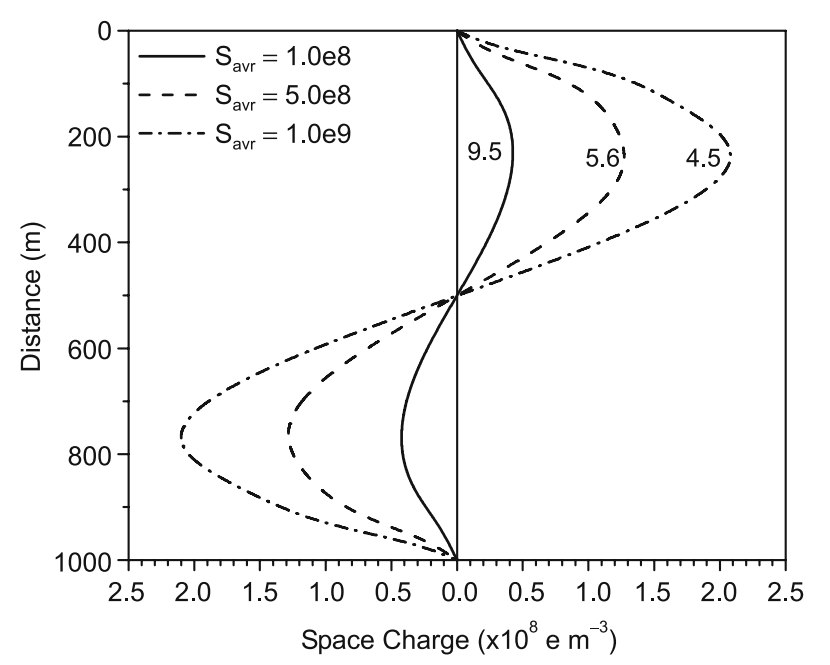

Figure 7. Variation in uni-polar space charge within the cloud having different drop concentrations at different sizes.

\subsection{Uni-polar space charge}

Regions of dominance of ions and charged particles create a gradient in the air conductivity in presence of non-zero current density, which lead to the presence of space charge. The atmospheric electrical conductivity is generally increased exponentially with altitude in fair weather condition. However, it decreases inside the cloud (and aerosol) layers where ions are scavenged by cloud drops (and aerosol particles), which are highly associated with electric field as discussed earlier, so the regions of net uni-polar space charge will often exist on the upper and lower surfaces of the layers (Harrison and Carslaw 2003).

The present simulation results the variations in uni-polar space charge within the cloud containing different drop concentrations at different sizes, which are shown in figure 7 . The average net positive and net negative space charges $\left(\sim 2.6 \times 10^{7} \mathrm{e} \mathrm{m}^{-3}\right)$ were observed at the top and bottom of the cloud, which contains average drop concentrations $1.0 \mathrm{e} 8 \mathrm{~m}^{-3}$ of radii $\sim 9.5 \mu \mathrm{m}$. The space charge was observed to be increased by $\sim 3$ and 5 times as the average drop concentrations increased by 5 and 10 times with significant decrease in drop radii $\sim 41 \%$ and $52 \%$, respectively. Both positive and negative space charges were observed to be maximum at $\sim 250$ and $750 \mathrm{~m}$ altitude level, respectively inside the cloud (from its top) containing different drop concentrations of different sizes.

\section{Discussions and implications to the natural cloud}

An attempt has been made to develop a numerical model for the production of space charge and to understand the behaviour of other electrical parameters within the stratiform cloud. Though the present simulated results show some discrepancies to the natural conditions, which could be due to simulations made under some basic assumptions and limitations to solve the complex non-linear coupled differential equations, it is crucial to study these electrical parameters to understand their impacts on non-thunderstorm clouds, and therefore conceivably on climate, via cloud microphysical processes (Harrison 2005).

In an earlier study, Zhou and Tinsley (2007) also modeled the production of space charge and its partition between charges on droplets, aerosol particles and ions and perform its sensitivity for the variations of different electrical parameters. They have considered mono-dispersive aerosol size distribution, with at most a single positive and negative charge on aerosol. They have also considered the droplet concentrations of mono-disperse size distribution and reported typically 50-100 elementary charges on both positively and negatively charged droplets. Though most of the attachment of ions within the clouds occurs on droplets rather than on aerosol particles, we have considered only droplet concentrations of mono-disperse size distribution having single positive and negative charges in the present study. A discussion on the implications of assumptions considered in the present study to the natural condition have been given in the next section by comparing simulated results with those obtained by Zhou and Tinsley (2007) using a cloud model and also with few available observational results.

\subsection{Effects of single and multiple droplet charges}

Enhancement in the concentration of cloud droplets within cloud increases the ions attachment, which reduces the ion concentrations and results in an increase in the charged drop concentrations within the cloud as clearly seen in figures 4 and 5, respectively. A decrease in the ionic concentrations reduces ionic conductivity within the cloud which, in turn, results in an increase in electric field (figure 6). The above results help in producing positive and negative space charges at top and bottom boundaries of the cloud, respectively, which increases significantly with an increase in cloud drop concentrations (figure 7). The magnitude of the above results differs significantly from the results obtained by Zhou and Tinsley (2007, figure 3), which is clearly a consequence of considering mono-disperse size distributions of single positive and single negative charged aerosols along with multiple charged droplets in a cloud model having comparatively less 
cloud layer thickness. Nevertheless, the nature of variations in the electrical parameters was found to be nearly similar to the present simulated results. To understand the percentage difference in the magnitude of the electrical parameters obtained from the present simulation and simulation done by Zhou and Tinsley (2007), a comparison in the magnitude of electric field was done for an identical case for the droplet concentrations of $1.0 \mathrm{e} 8 \mathrm{~m}^{-3}$. The simulated electric field value in the present study shows a decrease of $\sim 30 \%$ from the value obtained by Zhou and Tinsley (2007).

In an earlier study, Griffiths et al (1974) have shown that the effect of droplet charge (varied over a wide range) and its distribution over the droplets on normalized conductivity (the ratio of the in-cloud value to the clear-air value at the same altitude) is significantly very small in the weakly-electrified clouds. However, the only significant effect exercised by cloud charge is to introduce an asymmetry into the process of ionic immobilization. Zhou and Tinsley (2007) showed that $50 \%$ variations in air-Earth's current density $\left(J_{z}\right)$ can cause up to about $40 \%$ variations in charge on droplets and aerosols. These variations can certainly influence the collision processes between them and may affect cloud cover and climate. Earlier, Tinsley et al (2006) have numerically demonstrated that charges on aerosol particles and/or cloud droplets modify the droplet-particle collision efficiencies involved in scavenging, and the droplet-droplet and particle-particle collision efficiencies involved in coalescence of droplets and particles, even in weakly-electrified clouds and aerosol layers. Apart from the model estimated results, there are also a few observational evidences for the enhancement of space charge within the non-thunderstorm cloud. In the earlier studies, Clark (1958); Imyanitov and Chubarina (1967) have observed the space charge density typically $\sim 0.5-1.0 \times 10^{8} \mathrm{e} \mathrm{m}^{-3}$, which are nearly comparable to the present model estimated values. On the other hand, Tinsley (2000) estimated the space charge density at the interface of top of the cloud and found very large magnitude of charge density $\left(\sim 10^{9}-10^{10} \mathrm{e} \mathrm{m}^{-3}\right)$, which are due to his assumption of a very thin region $(\sim 1-10 \mathrm{~m})$ above the cloud where space charge accumulation occurs (Harrison and Carslaw 2003).

The discrepancy in the present study of space charge density and the other electrical parameters, apart from the layer thickness, could also due to the electrical parameter's dependence on several factors, including the stability of cloud layer in terms of turbulent mixing (not included in the present model simulation), ionization rate $(q)$ and the total air-Earth current density $\left(J_{z}\right)$ (Harrison and Carslaw 2003), which are considered
Table 3. Variation in space charge within the cloud at varying cloud thickness.

\begin{tabular}{cc}
\hline $\begin{array}{c}\text { Cloud thickness } \\
(\mathrm{m})\end{array}$ & $\begin{array}{c}\rho_{+}\left(\rho_{+_{\mathrm{Max}}}\right) \\
\left(\mathrm{e} \mathrm{m}^{-3}\right)\end{array}$ \\
\hline 1000 & $8.07 \mathrm{e} 7(1.27 \mathrm{e} 8)$ \\
500 & $1.61 \mathrm{e} 8(2.45 \mathrm{e} 8)$ \\
100 & $5.85 \mathrm{e} 8(7.64 \mathrm{e} 8)$ \\
50 & $7.77 \mathrm{e} 8(1.0 \mathrm{e} 9)$ \\
\hline
\end{tabular}

as constant under steady condition and taken as the typical values (table 1) for calculating initial boundary conditions of the model. Apart from the above factors, cloud drop concentrations and their sizes are the other important parameters that can also profoundly affect the various electrical parameters within the cloud, which is not mentioned in the earlier studies (Clark 1958; Imyanitov and Chubarina 1967; Tinsley 2000) and considered slightly different from the present simulations by Zhou and Tinsley (2007) in the cloud model. It can change the total ionic and charged drop concentrations and thus modulate the total space charge density by changing electric field within the cloud.

\subsection{Effects of thickness of cloud layer}

The model presented here is for the stable cloud layer. However, localized downward (and upward) winds near the top (and bottom) of the cloud can cause narrowing of the cloud layer and thus increased gradient of all the electrical parameters within the cloud (Zhou and Tinsley 2007). Zhou and Tinsley (2007) have reported that variations in vertical air-Earth's current density $\left(J_{z_{1}}\right.$ and $\left.J_{z_{2}}\right)$ due to variations in relative amounts of positive and negative ion concentrations $\left(n_{1}\right.$ and $\left.n_{2}\right)$ create space charges at the cloud boundaries. Consequently, more positive ions can flow downward than the negative ions flow upward from the top of the cloud, because of the decreased ion concentration within the cloud. Similarly, more negative ions can flow upward from the bottom of the cloud than positive ions flow down.

To understand the dependence of space charge on thickness of cloud layer, simulations have been done for the variations of space charge within the cloud by varying cloud thickness. Simulations were done for a single case of cloud, containing droplet concentrations of $5.0 \mathrm{e} 8 \mathrm{~m}^{-3}$ having drop radii of $\sim 5.6 \mu \mathrm{m}$. Results are shown in table 3 where the values outside the brackets indicate net average positive space charge at top of the cloud; the values in the brackets indicate maximum positive space charge within the cloud. Similar magnitude with opposite sign, i.e., negative space charge, was 
observed at bottom of the cloud. Table 3 clearly reveals that the magnitude of space charge highly depends on cloud layer thickness. A significant increase in space charge with narrowing cloud layer thickness was observed in the present simulation. Nearly similar feature in the nature of variation of space charge within the cloud was reported earlier by Tinsley (2000); Harrison and Carslaw (2003); Zhou and Tinsley (2007). Other electrical parameters within the cloud except electric field show similar behaviour as discussed above for space charge. However, a significant decrease in electric field was observed with decrease in cloud thickness as can be understood from equation (18).

\section{Conclusions}

- Numerical simulations of the production of space charge and the variations in other electrical parameters within the low level stratiform cloud have been done by assuming mono-disperse droplet distribution, with at most a single positive and/or negative charge.

- The magnitude of average positive ion concentrations was observed to be more than the average negative ion concentrations within the cloud due to low scavenging rate of positive ions than the negative ions, which is highly attributed to their mobilities. Consequently, low concentration of average positive charge drops were observed as compared to the average negative charge drops. On the other hand, the concentration of average net positive ions and net positively charged drops were observed to be higher near the top of cloud than the concentration of average net negative ions and net negatively charged drops; however, reverse was observed near the cloud bottom.

- The magnitude of maximum electric field $\left(E_{\max }\right)$ was observed $\sim 260 \mathrm{~V} \mathrm{~m}^{-1}$ at middle of the cloud having average drop concentrations $1.0 \mathrm{e} 8 \mathrm{~m}^{-3}$ with radii $\sim 9.5 \mu \mathrm{m}$, which increases significantly by $\sim 3$ and 5 times as the average drop concentrations increased by 5 and 10 times with subsequent decrease in drop radii $\sim 41$ and $52 \%$, respectively. However, the magnitude of minimum electric field $\left(E_{\min }\right)$ was observed to be constant $\left(\sim 27 \mathrm{~V} \mathrm{~m}^{-1}\right)$ at both the edges of cloud for any drop concentrations.

- Net positive space charge density was observed at the top and negative at the bottom of the cloud, which was attained $\sim 2.6 \times 10^{7} \mathrm{e} \mathrm{m}^{-3}$ when cloud containing the average drop concentrations $1.0 \mathrm{e} 8 \mathrm{~m}^{-3}$ having drop radii $\sim 9.5 \mu \mathrm{m}$. The space charge density was observed to be increased by $\sim 3$ and 5 times as the average drop concentrations increased by 5 and 10 times with significant decrease in drop radii $\sim 41$ and $52 \%$, respectively.

- All the above electrical parameters are highly sensitive to the thickness of the cloud. Except electric field within the cloud, other electrical parameters show significant increase with narrowing the cloud thickness. However, a significant decrease in electric field was observed with decrease in cloud depth.

\section{Acknowledgements}

This work was supported through a grant from ISRO RESPOND program. The authors thank Prof. B A Tinsley from the University of Texas at Dallas, Richardson, Texas, USA for his valuable discussions/suggestions in constructing the equations for this study. Authors are also thankful to Dr Devendraa Siingh from Indian Institute of Tropical Meteorology, Pune for his valuable suggestions. Authors are grateful to the anonymous reviewer for the constructive and valuable comments/suggestions, which helped to improve the scientific content of the manuscript.

\section{References}

Altaratz O 2003 The electrical development in thunderclouds in the eastern Mediterranean; Ph.D. thesis, Tel Aviv University.

Anderson T L, Covert D S and Charlson R J 1994 Cloud droplet number studies with a counterflow virtual impactor; J. Geophys. Res. 99 8249-8256.

Carslaw K S, Harrison R G and Kirkby J 2002 Cosmic rays, clouds, and climate; Science 298 1732-1737.

Chalmers J A 1967 Atmospheric electricity, 2nd edn, Pergamon, Oxford.

Chiu C S 1978 Numerical study of cloud electrification in an axisymmetric, time-dependent cloud model; J. Geophys. Res. 83 5025-5049.

Chiu C and Klett J D 1976 Convective electrification of clouds; J. Geophys. Res. 81 1111-1124.

Clark J F 1958 Fair weather atmospheric electric potential and its gradient; In: Recent advances in atmospheric electricity (eds) L G Smith (New York: Pergamon) pp. $61-73$.

Farrar P D 2000 Are cosmic rays influencing oceanic cloud coverage or is it only El Nino; Climate Change 47 7-15.

Ghoshdastidar P S 1998 Computer simulation of flow and heat transfer (New Delhi: Tata McGraw-Hill Publishing Co. Ltd.) 295p.

Gillani N V, Schwarts S E, Leaitch W R, Strapp J W and Isaac G A 1995 Field observations in continental stratiform clouds: Partitioning of cloud particles between droplets and unactivated interstitial aerosols; J. Geophys. Res. 100 18,687-18,706.

Griffiths R F, Latham J and Myers V 1974 The ionic conductivity of electrified clouds; Quart. J. Roy. Meteor. Soc. 100 181-190.

Gringel W, Rosen J M and Hoffman D J 1986 Electrical structure from 0 to 30 kilometers: The Earth's electrical environment, (Washington, D.C.: NAS Press) pp. $166-182$. 
Harrison R G 1992 Aerosol charging and radioactivity; Ph.D. Thesis, The University of London.

Harrison R G 2005 The global atmospheric electrical circuit and climate; Surv. Geophys. 25(5-6) 441-484.

Harrison R G and Carslaw K S 2003 Ion-aerosol-cloud processes in the lower atmosphere; Rev. Geophys. 41(3) $2-1-2-26$.

Helsdon J H Jr, Wojcik W A and Farley R D 2001 An examination of thunderstorm charging mechanisms using a two-dimensional storm electrification model; J. Geophys. Res. 106(D1) 1165-1192.

Kirkby J 2007 Cosmic rays and climate; Surv. Geophys. 28 333-375.

Imyanitov I M and Chubarina E V 1967 Electricity of the free atmosphere; Isr. Program for Sc. Transl., Jerusalem.

Jain M K 1984 Numerical solution of differential equations (2nd edn), Wiley Eastern Limited, New Delhi, India.

MacGorman D R and Rust W D 1998 The electrical nature of storms, Oxford University Press, Oxford.

Marsh N and Svensmark H 2000 Low cloud properties influenced by cosmic rays; Phys. Rev. Lett. 85(23) 5004-5007.

Masuelli S, Scavuzzo C M and Caranti G M 1997 Convective electrification of clouds: A numerical study; J. Geophys. Res. 102(D10) 11,049-11,060.

Mohnen V A 1977 Formation, nature and mobility of ions of atmospheric importance; In: Electrical Processes in Atmospheres (eds) H Dolezalek and R Reiter (Darmstadt, Germany: Steinkopff-Verlag).

Mouel J et al 2005 On long-term variations of simple geomagnetic indices and slow changes in magnetospheric current: The emergence of anthropogenic global warming after 1990? Earth Planet. Sci. Lett. 232273.

Pruppacher H R and Klett J D 1997 Microphysics of clouds and precipitation; 2nd revised edn (Dordrecht: Kluwer Academic Publishers) Chapter 18.

Rust W D and Moore C B 1974 Electrical condition near the bases of thunderclouds over New Maxico; Quart. J. Roy. Meteor. Soc. 100 450-468.
Saunders C 2008 Charge separation mechanisms in cloud; Space Sci. Rev. 137 335-3538.

Schonland B F J 1953 Atmospheric Electricity, 2nd edn, Methuen, New York.

Siingh Devendraa and Singh R P 2009 The role of cosmic rays in the Earth's atmospheric process; PramanaJ. Phys. 74(1) 153-168.

Siingh Devendraa et al 2007 The atmospheric global electric circuit: An overview; Atmos. Res. 84 91-110.

Solanki S K 2002 Solar variability and climate change: Is there a link? Astro. Geophys. 43 5.9-5.13.

Svensmark H and Christensen E F 1997 Variation of cosmic ray flux and global cloud coverage - a missing link in solar climate relations; J. Atmos. Solar Terr. Phys. 59 $1225-1232$.

Tinsley B A 2000 Influence of solar wind on the global electric circuit, and inferred effects on cloud microphysics, temperature, and dynamics in the troposphere; Space Sci. Rev. 94 231-258.

Tinsley B A, Rohrbaugh R P, Hei M and Beard K V 2000 Effect of image charges on the scavenging of aerosol particles by cloud droplets, and on droplet charging and possible ice nucleation processes; J. Atmos. Sci. $\mathbf{5 7}$ $2118-2134$.

Tinsley B A and Yu F 2004 Atmospheric ionization and clouds as links between solar activity and climate; $A G U$ Monograph: Solar variability and its effect on climate $\mathbf{1 4 1}$ 321-339.

Tinsley B A, Zhou L and Plemmons A 2006 Changes in scavenging of particles by droplets due to weak electrification in clouds; Atmos. Res. 79 266-295.

Yu F and Turco R P 2001 From molecular cluster to nano-particles: Role of ambient ionization in tropospheric aerosol formation; J. Geophys. Res. 106(D5) 4797-4814.

Zhou L and Tinsley B A 2007 Production of space charge at the boundaries of layer clouds; J. Geophys. Res. 112(D11203) doi: 10.1029/2006JD007998. 